\title{
Feature Structure Based Semantic Head Driven Generation
}

\author{
Gen-ichiro KIKUI \\ ATR Interpreting 'Telephony Research Laboratories \\ 2-2 Hikari-dai, Seika-cho, Soraku-gun, Kyoto 619-02 JAPAN \\ kikui@atr.la.atr.co.jp
}

\begin{abstract}
This paper proposes a generation method for feature-structure-based unification grammars. As compared with fixed arity term notation, feahure structure notation is more flexible for representing knowledge needed to generate idiomatic structures as well as general constructions. The method enables feature structure retrieval via multiple indices. The indexing mechanism, when used with a semantic head driven generation algorithm, attains efficient generation even when a large amount of generation knowledge must be considered. Our method can produce all possible structures in parallel, using structure sharing among ambiguous substructures.
\end{abstract}

\section{Introduction}

Practical generation systems must have linguistic knowledge of both specific expressions like idioms and general grammatical constructions, and they should efficiently produce surface strings applying that knowledge [1][2].

In order to satisfy the first requirement, on system employs a set of trees annotated with feature structures to represent generation knowledge. Gach tree represents a fragment of a syntactic structure, and is paired with a semantic feature structure. We can describe idiomatic constructions, by making a tree which contains lexical specifications and is paired with a specific rather than general semantic structure. Because feature structures allow partial specification, we can encode generation knowledge ranging over unultiple levels of generality in a uniform way.

However, notice that this property will be restricted if we use DCG or (fixed arity) term notation 1. Suppose there is a generation kinowledge structure whose syntactic part is "go on foot". The feature structure notation of its semantic part will be something like:

\footnotetext{
The flexibility of structure notation compared to term notation is also discussed in [4].
}

\author{
[Reln GO] \\ [Agent ?agent []] \\ [Instrument FOOT]]. \\ (1)
}

while the term notation is :

$$
\text { instrument(go(Agent), foot) (2) }
$$

'These two notations seem to be equivalent, but there is a crucial differeuce. A generation knowledge structure containing the feature-based semantics will still be unifiable cven if the semantic input to be unified contains additional material. Thus the knowledge structure will be discovered and its syntactic information can be used for generation. By contrast, a term-based input with additional elements woud not unify with the term-based semantic structure shown above. It would thus be necessary to create arlditional generation structures containing distinct (though partly overlapping) term-based semantic structures. Such additional structures are redundant and cause superfluons output.

for example, consider the augmented feature structure

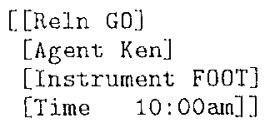

It will indeod unify with (1) above. But termbased input semantic structure (4) will not unify with term-based semantic structure (2).

instrument (time $(\mathrm{go}(\mathrm{ken}), 10: 00 \mathrm{am})$, foot).

To unify (2), semantic structure (5) would also be recuiresl.

time(instrument (go(ken), foot), 10:00an). 
For this reason, our generation knowledge consists of trees represented as feature structures. A tree can be substituted for a leaf node of another tree to form a larger structure. 'Thus, the tree can be regarded as a rule in a context-free feature-structure-based unification grammar.

The second requirement for a generation system is efficient creation of syntactic structures. 'This is the main topic of this paper. Our system is based upon Semantic Head Driven Gencration [6], which is an efficient algorithm for unification based formalisms. However, this algorithm requires some additional mechanisms to efficiently retrieve relevant generation knowledge, because feature structures can not be easily indexed.

The algorithm presented here uses a multiple index network of feature structures to efficiently choose relevant generation knowledge from the knowledge base. The algorithm also uses an hypothetical node so as to efficiently maintain ambiguous structures during generation.

\section{Phrase Description(PD)}

Generation knowledge is represented as a set of trees annotated with feature structures. liach tree is called a Phrase Description (PI).

An example of a P'D is shown in Figure.l.

Structure:

Annotation:

(S AUX (NP PRON) VP)

$\begin{aligned} \text { (S [ syn } & {[\text { cat S] [inv +] ] }] } \\ \text { [sem }[ & {[\text { reln REQUEST] }} \\ & {[\text { agen *SP*] }} \\ & {[\text { recp *HR*] }} \\ & {[\text { obje ?ACTION] ]]] }) }\end{aligned}$

(AUX $[[\operatorname{syn}[$ [Cat AUX] [1ex "would"] [v-morph PAST $]]]])$

(NP [[syn [[cat NP] [case NOM]]]])

(PRON [ [syn [ [cat PRON] [case NOM] [lex "you"]]]])

(VP [[syn [[cat VP] [v-morph BSE]]] [sem ?ACTION]])

Figure 1: an example of a $\mathrm{Pl}$

A PD consists of two parts: a structure clefinition and feature structure annotation (Structure and Annotation in Figure 1).

The structure definition defines the structure of a tree by using a list in which the first element corresponds to the mother node and the rest of the elementis correspond to daughters. lach daughter may be a tree rather than a simple node.
The annotation part specifies the feature structure of each symbol appearing in the structure definition. A feature structure description can contain tags or variables (symbols with "?" as a prefix in the figure). 'The scope of a tag in a PD is the entire PD.

Each node should have a semantic and syntactic feature structure. The semantic feature on the root node of a $\mathrm{PD}$ represents the semantics of the PD; thus we call it the semantic structure of the PD.

Although the description represents a tree, it is the same as for a (partial) derivation structure of a unification-based CFG, because the current system does not allow adjoining operations. If the structure definition of every $P D$ is restricted to mother-daughter relations only, the PD set is strictly equivalent to a unification-based CFG.

\section{Generation Algorithm}

Our algorithm is an efficient extension of Semantic Head Driven Generation. The major extensions are: 1) it handles feature structures directly, and 2) it creates all possible phrase structures in parallel. These extensions are embodied mainly in the PI) activation and ambiguity handling mechanisms discussed in this section.

\subsection{Overview of the algorithm}

The main part of the generation process is expansion process, which iterates through expanding node selection, activation, precombination, and application, using an expanding node agenda.

Input to the process is a feature structure containing syntactic, semantic and pragnatic features as an initial constraint on the root node.

The expanding node agenda contains the unlexicalized leaf nodes of the tree under creation. At the beginning of the process, it contains only one node, which has the feature structure given as an initial constraint.

The expanding node selection step picks up one node, say expanding node, from the agenda. If no norle is picked up, the expansion process stops.

The PD activation step activates all P'D's whose semantic structures sabsume the semantic structure of the expanding node.

The precombination stej makes PD sequences from activated $P D$ 's to satisfy some constraints.

The application step instantiales the PD sequence(s) and applies it to the expanding node. 


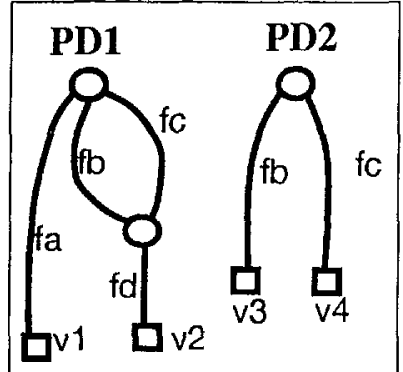

Figure 2: an example of dags

It also pushes unlexicalized leaf nodes into the expanding node agenda.

\subsection{Expanding Node Selection}

The expanding node selection step is for fetching one node from the expanding node agenda. From among the nodes whose semantic feature has been instantiated, one is chosen. In this step, if the fetched node satisfies some termination conditions (if, for instance, it satisfies the conditions for slash termination), the node is discarded (i.e., not expanded any more). If the agenda is empty or contains no node with an instantiated semantic feature, the expansion process stops.

\subsection{Activation}

This step is responsible for selecting all PID's whose semantic structures subsume the semantic structure of an expanding norle. 'The selection is done by traversing a multiple index network of PD's called the PD net.

\subsubsection{Compiling PD's}

A set of PD's are pre-compiled into a PD net. Suppose there are two PD's whose semantic structures $^{2}$ are defined as the dags (i.c. $d i$ rected acyclic grophs) in Figure 2. In the figure, $f a, f b, f c, \ldots$ and $v 1, v 2, \ldots$ represent arc labels (feature names) and atomic values respectively. These PD's are compiled to the PD net shown in Figure 3.

The net has two kinds of nodes: path nodes $\left(p_{i}\right)$, and $P D$ nodes $\left(P D_{j}\right)$. These nodes are linked by three kinds of labeled directed arcs:

\footnotetext{
${ }^{2}$ The semantic feature of $a P D$. is a semantic feature on the root node of the PD
}

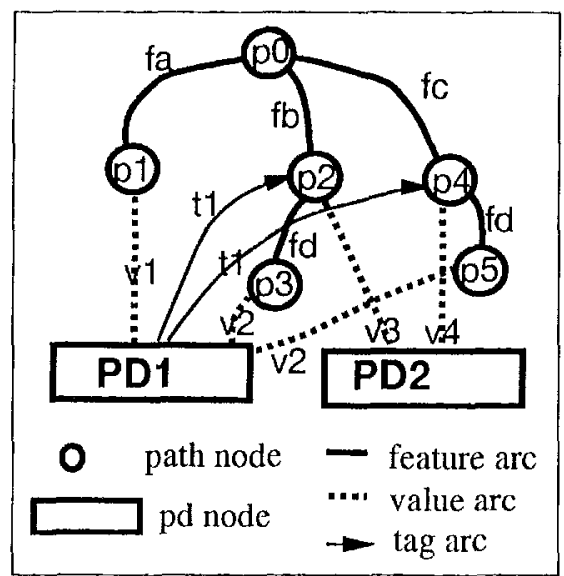

Figure 3: an example of $\mathrm{PD}$ net

feature arcs(bold lines), value arcs(dashed), and tag arcs(with arrows).

A path node is used to represent a particular feature path in one or more feature structure.

As shown in Figure 3, path nodes are linked by bold feature arcs to form a tree. The sequence of arc labels from the root path node to a path node $p_{i}$ is the path of $p_{i}$. In Figure $3, \mathrm{p} 3$ and $\mathrm{p} 5$ show paths ( $f b$ fd) and ( $f c f d$ ) respectively.

Each PD node (rectangle) corresponds to a particular PD, which may have value arcs and tag arcs.

- Value Arcs: Which PD's contain which atomic values along certain paihs?

A PD node may be linked to path nodes with value ares.

If a (rectangular) PD node is linked to a (round) path node pn with a dashed value arc labeled $\mathrm{v}$, then following the path leading to pn yields atomic value $v$ in that $P D$.

Consider the dashed value arc vl in Figure 3. It indicates that following path fa in $P D_{1}$ yields an atomic value $\mathrm{v} 1$. This is just the situation depicted in Figure 2.

- 'Tag Arcs: In a given P'D, which paths share a. certain feature structure as a value?

A PD node may also be linked to path nodes with tag ares.

If two tang arcs have the same label and they connect a $\mathrm{PD}$ node to two path nodes, say pn 1 and pn2, then the feature structure of that PD has a substructure which is the value of both paths, that of pn1 and pn2. 
For example, the two tag arcs from rectangular PD1. node labeled "t 1 " in Figure 3 show that the semantic structure of PD1 has a substructure serving as the value of $(\mathrm{fb})$ and $(\mathrm{fc})$.

\subsubsection{Traversing the PD net}

The data structure of nodes and arcs are shown itl Figure 4.

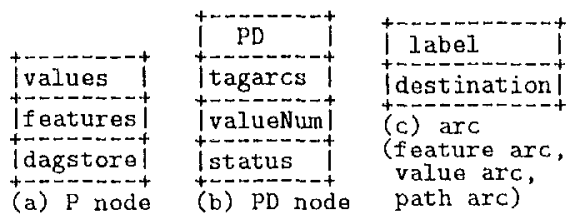

Figure 4: Node and Arc structures

A path node has three slots: values, features, and dagstore. The values slot and the arcs slot contain value arcs and feature arcs respectively. The dagstore slot is initially empty; later it holds a pointer to a dag which passed the path node.

Each $P D$ node has a $P D$ slot, a tagarcs slot, a valueNum slot, and a status slot. The I'D slot contains a pointer to the represented PD itself. The tagarcs slot contains the data structure of the tagarcs (see below). The valueNum slot has the number of value arcs to the PD node. Fior example, the value of the number slot of PJI node in Figure 3 is 3, becanse the node has one value arc labeled $\mathrm{v} 1$ and two value arcs labeled v2. The status slot holds integer and is initially set to 0 .

Every type of arc has two slots, 'label' and 'destination'. 'Label' is an atomic symbol which labels the arc, and 'destination' is a pointer to the node which is the destination of the arc.
We use the PD net as a dataflow net. The en* try point of the net is the root path node and the token which flows is a dag of a semantic feature structure.

The action of a path node is shown in Figure 5. "fajlure" means there is no PD whose semantic structure subsumes the given dag. Thus the entire retrieval process fails.

The action of a PD node is shown in Figure 6. The status is incremented each time the node receives a token. $\Lambda$ s a result, if all atomic values in the semantic structure of the PI) are satisfied, the status becomes equal to the valueNum (that is the number of atomic values). Once this is detected, then unifiability of shared structure is checked by calling the tagtest procedure.

Tagtest tests unifiability of the dags in the dagstores of $p($ ath $)$ nodes connected by tag ares with the same label. In Figure 3, if the status of PDI becomes 3 and if the dag in $p 2$ and the dag in $p 4$ are identical, then the I'D becomes active. That is, the PD has been found to subsume the generation input. It may or may not actually be applied, depending on later events.

$$
\begin{aligned}
& \text { PROCEDURE PDnodeAction(pdNode, value) } \\
& \text { pdNode.status - pdNode.stalus }+1 ; \\
& \text { IF pdNode.status = pdNode.valueNum } \\
& \text { and tagtest(pdNode.tagarcs) = T THEN } \\
& \text { activate(pdNode.PD); }
\end{aligned}
$$

Figure 6: Procedure of a PD node

If there is a PI) node whose valueNums is 0 (i.e. No atomic value is specified in the semantic structure), node action of the PD node is in volied after dataflow is terminated.

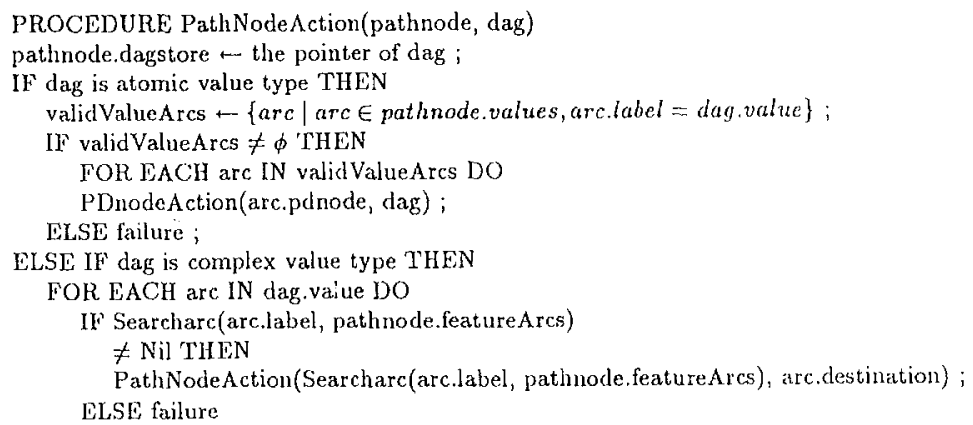

Higure 5: Procedure of a path node 


\subsection{Precombination}

The precombination step is responsible for making sequences of PD's from activated PD's under certain constraints. A PD sequence is a rough plan for a potential structure which can realize a semantic specification of the node being expanded ${ }^{3}$. If no sequence is obtained, the ambiguity resolution process, discussed later, is invoked.

We divide PD's into two groups: propagation type and non-propagation type. A propagation type $\mathrm{PD}$ has has one propagation node. $\mathrm{A}$ propagation node of a PD is a leaf node whose semantic structure is identical with the semantic structure of the root node of the PD ${ }^{4}$. The rest of the PD's, which have no propagation nodes, are classified as non-propagation type PD's. This distinction is an extension of Shieber's chain rule and non-chain rule distinction.

A PD sequence $P D_{1} \ldots . . P D_{n}$ must satisfy the following constraints.

1. semantic structure sharing constraints

(a) $P D_{i}(1 \leq i<n)$ is a propagation $P D$,

(b) $P D_{n}$ is a non-propagation $\mathrm{PD}$,

Under these constraints, the system can make a partial phrase structure by unifying the propagation node of $P D_{i}$ with the root node of $P D_{i+1}$. The root node of the created structure contains the unified semantic structure of all semantic structures of PD's in the sequence.

\section{2. local unifiability constraints}

(a) the root node of $P D_{1}$ is unifiable with the expanding node

(b) $P D_{i}$ and $P D_{i+1}$ are connectable

where $P D_{i}$ is conncctable to $P D_{j}$ if $P D_{i}$ is a propagation $\mathrm{PI}$ ), and the propagation node of $P D_{i}$ is unifiable with the root node of $P D_{j}$.

These constraints are necessary conditions for unifiability throughout the entire PD sequence, which is called the global unifiability of a $P D$ sequence. In contrast to such global unifiability constraints, the local unifiability can be pre-computed, or compiled, before generation process.

\footnotetext{
${ }^{3} \mathrm{~A}$ PD sequence is roughly corresponds to a bottom-tup chain in [0]

'Our current system does not allow 'D's with multiple semantic hearl
}

\section{3. covering constraint}

Let $\mathrm{fs} 1$ be the unified semantic structure of all semantic structures of PD's in the sequence. Fs 1 must contain every top feature of the semantic structure of the expanding node, where a top feature of a feature structure is defined as a top-level feature name.

The covering constraint ensures complete generation [6]. If the constraint is not satisfied, a given semantic structure may not be completely realized in the generation result. For example, if an input semantic strucure is (3) (in Section 1) and the unified semantic structure of a PI) sequence is (1), then the resulting $P D$ sequence lacks the locative phrase for the "time" feature, which will not appear in the generation result.

4. disjointness constraints

For each $\mathrm{PD}\left(P D_{i}\right)$, there is no other PD $\left(P D_{j}(i \neq j)\right)$, such that $P D_{i}$ has a top arc whose label is included in the set of top arcs of $P D_{j}$. The definition of top arc is given above.

If this constraint is not satisfied, the generation result may contain duplicated or invalid expressions. For example, if a PD sequence contains two distinct PD's each of which is for a locative adjunct and has a "time" feature on the top level, the generation result will have two locative adjuncts for one semantic feature (i.e. the "time" feature).

The disjointness constraint also ensures compact generation. Suppose a coherent and complete generator produces a string $w$, and the grammar assigns a scmantic structure fso to $w$ using a set of rules $R$. String $w$ is minimal if every sub-structure of $f s o$ is supplied from one rule in $R$. The generator is compact if any string $w$ is minimal.

In general, completeness, and compactness cannot actually be judged until the entire generation finishes. Thus the last two constraints ( 3 and 4) do not really guaran tee completeness and compactness; rather, they help to limit scarch space in a practical way.

\subsection{PD Application}

The PD application step is responsible for creating phrase structure(s) from $P D$ sequence(s) and attaching them to the expanding node. In this section, we restrict ourselves to the simple case 
such that there is only one $\mathrm{PD}$ sequence obtained during the previous step. The case of multiple $\mathrm{PI}$ sequences, (i.e., generation ambiguity), will be discussed in the next section.

First, the module connects all PD's in the $\mathrm{P}^{3} \mathrm{D}$ sequence $P D_{i} \ldots P D_{n}$ by unifying the propagation node of $P D_{i}$ with the root node of $P D_{i+1}$. All unification operations are quasi-destructive, or temporal [7]. The result of the unification is valid until the module copies it (see below).

If this entire unification process succeeds (i.e., if every PD in the sequence can indeed be unified, and the sequence thus proves to be globally unifiable; see 3.7), then the module makes a copy of the unified PD sequence. Otherwise expansion failure (see next section) is signified. The copy, which is a phrase structure tree, is called an instance of the PD sequence.

Then the module attaches (unifies) the instantiated PI sequence to the expanding node.

Finally, the system stores in the expanding node agenda leaf nodes of expanded structures which have no lexical feature values. .

\subsection{Ambiguity Handling}

\subsubsection{Ambiguity packing}

If multiple PD sequences are applicable to an expanding node, the substructure of the expanding node can not be uniquely determined, because each PD sequence indicates only an hypothesis for the potential substructure.

The system maintain these hypotheses in a special hypotheses siot on the undetermined expanding node.

For each PD sequence, a copy of the expanding node called an hypothesis node is created. 'Those copies are stored into the hypotheses slot of the original expanding node. Then the system applies each l'D sequence to the corresponding hypothesis node, as described in the previous section, and continues expansion.

In Pigure 7, three subtrees in the "hypo" slot on the undetermined node have been created for the hypothetical Pl) sequences.

The hypothetical PD sequences are not unified with the original expanding node, but unified with copies of the expanding node. This prevents the original feature structure of the undetermined node from being modified by further expansion of the hypothetical structures ('T-T3 in Figure 7 ).

The further expansion sometimes makes an hypothesis node inconsistent with the original

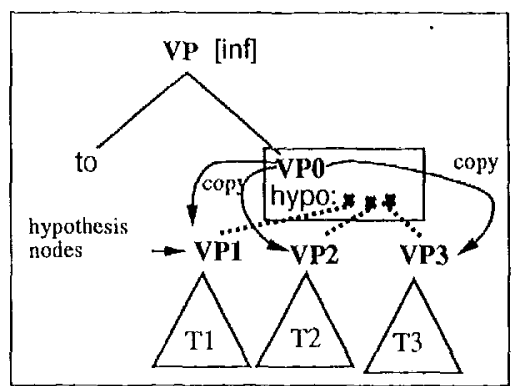

Figure 7: generation ambiguity

node. This is detected in the ambiguity resolution process described in the next section.

\subsubsection{Expansion Failure and Ambiguity Resolution}

Expansion failure occurs when:

1. Ilo $\mathrm{PD}$ is activated in the $\mathrm{PD}$ activation, or

2. no PD sequences are obtained in the precombination, or

3. no PD sequences satisfy global connectability in the application.

The failure signifies that the feature structure of the current expanding node is inconsistent with a set of Pl)'s given as generation knowledge.

The module searches for the nearest (i.e., lowest) liypothesis node $\left(N_{h}\right)$ dominating the failed expanding node and deletes $N_{h}$ from the hypotheses slot containing it.

If the number of hypothetical structures in the hypotheses slot of an undetermined node $\left(N_{u}\right)$ becones one, then $N_{u}$ and the root node of the remaining structure in the hypotheses slot are unified. If the unilication fails, ambiguity resolntion continues recursively upwird.

An exanple of ambiguity resolution is illustrated in Figure 8 . The values of the hypotheses slot of node $V P^{\prime}$ are the bypothetical norles VI'1, VP'2, and VP'3, corresponding to hypothetjcal trees $\mathrm{T} 1, \mathrm{~T} 2, \mathrm{~T} 3 \mathrm{r}$ 'spectively. If expansion failure occur in T'I and 'T'2, VI' 1 and VP'2 are removed from the hypothesis slot. Then, VP3 is unified with VI', because there is only one hypothesis node left in the slot $\mathrm{VI}^{\prime}$ node.

If there is no hypothesis node dominating the failed expansion node, the entire generation process fails. 


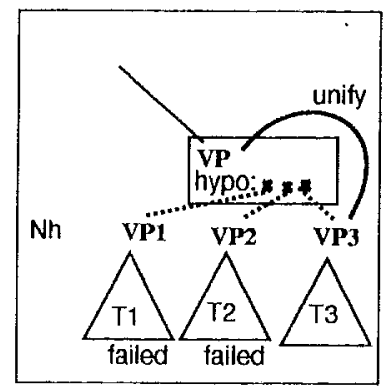

Figure 8: an illustration of generation ambiguity resolution

\subsection{Postprocess}

Expansion halts when no node is selected in the expanding node selection step. This does not necessarily mean the agenda is empty, because there may be some nodes without instantiated semantic structure.

How do such semantically empty nodes arise? The problem is that feature structures within hypothetical nodes are not allowed to unify with the feature structure on the "real" dominating node.

The solution is: for each hypotheticad node, we create a complete tree using copies of the "real" dominating structure, Feature structures can then be permitted to unify with dominating structures. Then, the system collects all unlexicalized leaf nodes as initial values of the expanding node agenda and starts the normal expansion loop again.

\section{Concluding Remarks}

A semantic head driven generation method based on feature structures is proposed in this paper. This method efliciently generates all possible phrase structures from a given semantic feature structure. 'The method involves multiple indexing of feature structures and a precombination mechanism. These mechanisms constrain applicable grammatical knowledge before instantiation; thus the method eliminates the copying of feature structures, which consumes computing resources.

The proposed grammar notation is appropriate for describing idiomatic phrase structures easily. 'To make the best use of the notation, we are extending the algorithm so that it can perform adjunct operation [9].

The algorithm is implemented in SL-Trans, a spoken language translation system [8].

\section{Acknowledgments}

The author would like to thank Mark Seligman for helpful comments on this paper and also would like to thank Akira Kurematsu, Tsuyoshi Morimoto and other members of ATR for their constant help and fruitful discussions.

\section{References}

[1] Hovy, E.H., "Generating Natural Language Under Pragmatic Constraints",Ph.D.Dissertation, Yale Univ., 1987

[2] Jacobs, P.S., "A generator for natural language in terfaces", In D.D.McDonald et al., editors, Natural Language Generation Systems, Chapter 7, Springer-Verlag, 1988

[3] Shieber,S. M., "An Introduction to Unification-Based Approaches to Grammar", CSLI, 1986

[4] Knight, K., "Unification: A Multidisciplinary Survey", ACM Computing Surveys, Vol.21, No.1, 1989

[5] Pollard,C. et al., "Information-based Syntax and Semantics Volume 1 Fundamentals", CSLI, 1987

[6] Shieber, S.M. et al., "A Semantic-HeadDriven Generation Algorithm", In Proceedings of 27 th ACL, 1989

[7] Tomabechi, II., "Quasi-Destructive Graph Unification", In Proceedings of 29 th $\mathrm{ACl}$, 1991.

[8] Morinoto, 'T., et al. " $\Lambda$ Spoken Language Translation System : SI,-TRANS2". In Proceedings of COLING'92, 1992.

[9] Vijay-Shanker, K. et al., "Feature Structure Based Tree Adjoining Grammars", in Proceedings of COLING's8, 1988 